\title{
BMJ Open Alcohol consumption and mortality in patients with mild Alzheimer's disease: a prospective cohort study
}

\author{
Sine Berntsen, ${ }^{1}$ Jakob Kragstrup, ${ }^{1}$ Volkert Siersma, ${ }^{1}$ Gunhild Waldemar, ${ }^{2}$ Frans \\ Boch Waldorff ${ }^{1,2,3}$
}

To cite: Berntsen S, Kragstrup J, Siersma V, et al. Alcohol consumption and mortality in patients with mild Alzheimer's disease: a prospective cohort study. BMJ Open 2015;5:e007851. doi:10.1136/bmjopen-2015007851

- Prepublication history and additional material is available. To view please visit the journal (http://dx.doi.org/ 10.1136/bmjopen-2015007851).

Received 2 February 2015 Revised 3 September 2015 Accepted 6 October 2015

\section{(1) crossank}

\section{${ }^{1}$ The Research Unit for} General Practice and Section of General Practice, Department of Public Health, University of Copenhagen, Copenhagen, Denmark ${ }^{2}$ The Research Unit for General Practice, Department of Public Health, University of Southern Denmark,

Odense C, Denmark

${ }^{3}$ Department of Neurology,

The Danish Dementia

Research Centre,

Rigshospitalet, University of Copenhagen, Copenhagen, Denmark

\section{Correspondence to} Dr Sine Berntsen; sineberntsen@hotmail.com

\section{ABSTRACT}

Objective: To investigate the association between alcohol consumption and mortality in patients recently diagnosed with mild Alzheimer's disease (AD).

Design: A post hoc analysis study based on a clinical trial population.

Setting: The data reported were collected as part of the Danish Alzheimer's Intervention Study (DAISY), a longitudinal multicentre randomised controlled study on the efficacy of psychosocial intervention in patients with mild AD across five county districts in Denmark.

Participants: 321 patients with mild AD (Mini-Mental State Examination $\geq 20$ ) were included. Data regarding current daily alcohol consumption were obtained from the patient's primary caregivers at inclusion.

Main outcome: All-cause mortality retrieved from The Danish Civil Registration System over a period of 36 months after baseline.

Results: Information about alcohol consumption was obtained from all 321 study participants: $8 \%$ were abstinent, $71 \%$ only had alcohol occasionally ( 1 or $<1$ unit/day), 17\% had 2-3 units/day and $4 \%$ had more than 3 units/day. An analysis adjusted for a range of potential confounders demonstrated a reduced mortality for patients with moderate alcohol consumption (2-3 units/day): HR 0.23 ( $95 \% \mathrm{Cl}(0.08$ to 0.69$)$ ) compared with patients who had 1 or $<1$ unit/ day. Mortality was not significantly different in abstinent patients or in patients with an alcohol consumption of more than 3 units/day, compared with patients drinking 1 or $<1$ unit/day.

Conclusions: In this cohort of patients with mild AD, moderate alcohol consumption (2-3 units/day) was associated with a significantly lower mortality over a period of 36 months. Further studies are needed in this area. These may especially focus on the association between alcohol consumption and cognitive decline in patients with $A D$.

\section{INTRODUCTION}

It is a well-established fact that excessive alcohol consumption has severe negative effects on both physiological and psychological health. Several population cohort
Strengths and limitations of this study

- Data in this study are disease-specific for Alzheimer's disease (AD). This gives us important knowledge on how a very common lifestyle factor such as alcohol affects the lives of people diagnosed with $A D$ specifically.

- Since extensive data were collected on each patient in the Danish Alzheimer's Intervention Study (DAISY) study, we were able to adjust for a wide range of potential confounders, which is essential when studying the effects of alcohol consumption.

- The results of this study are based on post hoc analysis, that is, the investigation of alcohol intake on mortality was not described in the original DAISY protocol.

- In the alcohol groups, other than our reference group (1 or $<1$ unit/day), the number of study participants was relatively low.

- The participants in this study have been specifically selected for an intervention study and there might therefore be an over-representation of better functioning patients. Patients with more severe disease, those with significant comorbidity and patients living in a nursing home without a primary caregiver might have other effects of alcohol.

studies suggest, however, that alcohol consumption may not be solely harmful. In fact, moderate alcohol consumption seems to have beneficial effects on various parts of our health, decreasing the risk of cardiovascular disease and mortality. ${ }^{12}$

Systematic reviews and meta-analysis on the association between alcohol consumption and dementia have concluded that at present there is no indication that light-to-moderate alcohol consumption is harmful to dementia. ${ }^{3}$ On the contrary, it has been stated that there is substantial evidence that light-to-moderate alcohol consumption reduces the risk of dementia and cognitive decline. ${ }^{4}$ 
While there are numerous studies focusing on alcohol as a risk factor for dementia and mortality in healthy subjects, virtually no attention has been paid to the effect of alcohol consumption in patients with Alzheimer's disease (AD).

It has been argued that social drinking may be harmful for patients with $\mathrm{AD} ;{ }^{5}$ however, we have not been able to identify any studies on the association between alcohol consumption and mortality in patients diagnosed with dementia. Considering that $\mathrm{AD}$ is a neurodegenerative disorder and that alcohol has known neurotoxic effects, one could easily jump to the conclusion that alcohol is damaging for patients with $\mathrm{AD}$. The aim of this study was to investigate whether the positive association between moderate alcohol intake and mortality shown in population-based studies on healthy subjects can be transferred to patients with mild AD.

\section{METHODS}

The data reported in this post hoc analysis were collected as part of the Danish Alzheimer's Intervention Study (DAISY), a longitudinal multicentre study on the efficacy of a psychosocial intervention in patients with mild AD in five county districts in Denmark. Details of the background, cohort and methods of the study have been reported earlier. ${ }^{6-8}$ Briefly, this multicentre, rater-blinded, randomised controlled study (RCT) included 330 outpatients with mild $\mathrm{AD}$, or dementia with Lewy bodies, and their 330 primary caregivers. The participants were randomised to control support during follow-up or to control support plus DAISY intervention (multifaceted and semitailored counselling, support and education) over a period of 12 months. The follow-up period was 3 years. The aim of the original DAISY study was to assess the efficacy at 12 months of an early psychosocial counselling and support programme for outpatients with mild $\mathrm{AD}$ and their primary caregivers. For the present study, only patients with $\mathrm{AD}(\mathrm{n}=321)$ were included.

\section{Participants}

Inclusion criteria for the patients were: (1) a Mini-Mental State Examination (MMSE) score $\geq 20$, (2) a clinical diagnosis within the past 12 months of probable $A D$ or mixed $A D$ with vascular components, (3) age $\geq 50$ and (4) having a primary caregiver willing to participate in the study. The patients met the Diagnostic and Statistical Manual of Mental Disorders, 4th Edition $^{9}$ criteria for dementia and the NINCDS-ADRDA $^{10}$ criteria for probable AD. Patients were excluded if they lived in a nursing home, participated in other intervention studies or if they had severe somatic or psychiatric comorbidity (including significantly impaired hearing or vision). Written informed consent to participate was obtained from all patients and primary caregivers.

\section{Assessment of alcohol intake}

At inclusion, the caregivers were asked about the patient's current average daily intake of alcohol.

The caregivers were asked in a questionnaire to assess: 'Alcohol consumption-current daily intake (tick off one box): no alcohol, only at parties, 1 or $<1$ unit/day, 2-3 units/day or more than 3 units/day'. Alcohol consumption was not divided into types of alcoholic beverages (beer, wine, spirits, etc).

Owing to the similar raw 3-year mortality $(18.4 \%$ and $20.2 \%$ ) and since the two classifications were overlapping, the groups 'only at parties' and ' 1 or $<1$ unit/day' were compiled under the title ' 1 or $<1$ unit/day'.

The Danish unit of alcohol was used in the study. A Danish unit of alcohol is $12 \mathrm{~g} / 15 \mathrm{~mL}$ of pure alcohol (a UK unit of alcohol is $10 \mathrm{~mL}$ of pure alcohol.)

\section{Assessment of mortality}

Mortality data were retrieved from The Danish Civil Registration System. ${ }^{11}$

\section{Assessment of confounders}

A number of potential confounders were recorded in order to adjust the analysis: age, sex, smoking, household status, MMSE, quality of life (QoL), awareness, education level and comorbidity.

MMSE was used to assess global cognitive functions. Scores ranged from 20 to 26 at inclusion, with higher scores indicating better cognitive performance. ${ }^{12}$

QoL (rated by the primary caregivers of the patient with $\mathrm{AD}$ ) was assessed with the quality of life Scale for $\mathrm{AD}$ (QoL-AD). ${ }^{13}$ This is a 13-item scale ranging from 13 to 52. Higher scores indicate a better QoL.

The Alzheimer's disease Cooperative Study activities of daily living (ADSC-ADL) scale was used to assess activities of daily living. It is a 23-item scale with a maximum score of 78. Higher scores indicate better function. ${ }^{14}$

On the basis of cognitive testing and interviews, the patient's level of awareness was rated with the Anosognosia Rating Scale, which is a categorical threepoint scale from Reed et al. ${ }^{15}$ Awareness was classified into 'full awareness', 'shallow awareness' and 'no awareness'.

Charlson Comorbidity Index (CCI) is a measure for the general mortality increase due to disease. ${ }^{16}$ Starting at the lowest score of zero, for each one of 19 different conditions a weight is added to the index if a hospital contact for this condition was recorded for a patient in the 3 years before the dementia diagnosis; more severe diagnoses had higher weights. Additionally, one point was added for each 10 years of age above 40 years.

Education level was recorded in six groups: no education, vocational, short education $(<3$ years), medium education (3-4 years), long education ( $>4$ years) and other.

The groups 'vocational', 'short education' and 'other' were collapsed into one category in our statistical analyses (=vocational/short). The same applies for 'Medium 
education' and 'long education' that were also collapsed into one category (=medium/long).

\section{Statistical analysis}

Baseline characteristics of the patients with various degrees of alcohol consumption were compared using Pearson's $\chi^{2}$ test for categorical variables and a nonparametric Kruskal-Wallis test for continuous variables.

The mortality associated with alcohol consumption was estimated by HR from a Cox regression model. The group 'one or less than 1 unit/day' was used as the index group. Regression analysis was performed both unadjusted and adjusted in a multivariable model for possible confounders (age, sex, MMSE, QoL-AD, ADSC-ADL, CCI, smoking, education and household status) to check whether apparent associations could be attributed to confounding.

Furthermore, the analysis was adjusted for the RCT allocation group to rule out that a previously observed increased mortality in the intervention group could be attributed to differences in alcohol consumption. ${ }^{6}$ Subgroup analyses into a possible difference between sexes, presence of comorbidity and whether the patient was living alone in the association of alcohol intake with mortality were tested by including an interaction term in the regression analyses. Furthermore, sensitivity analyses were performed omitting the patients reporting drinking alcohol 'only at parties', and omitting event in the first year of follow-up.

Statistical analysis was done using SAS V.9.4.

\section{RESULTS}

Information about alcohol consumption was obtained from all 321 study participants. Among these, $8 \%$ were abstinent, $71 \%$ only had alcohol occasionally ( 1 or less unit/day), $17 \%$ had 2-3 units/day and $4 \%$ had more than 3 units/day.

Table 1 shows the baseline characteristics of the study population according to categories of alcohol consumption. There were relatively more abstinent women than men and a higher percentage of men in the group of patients with higher alcohol consumption (more than 3 units/day). The number of 'never smokers' decreased with increasing alcohol consumption and there was a tendency towards people living alone generally drinking less than people living with others.

During the 36-month follow-up period, 53 (16.5\%) patients died. The effect of alcohol consumption on mortality is shown in table 2. The multivariable analysis demonstrated a reduced mortality for patients with moderate alcohol consumption (2-3 units/day): HR 0.23 (95\% CI (0.08 to 0.69$) \mathrm{p}=0.0076$ ) compared to patients who only had alcohol occasionally ( 1 or $<1$ unit/day). Abstinent patients or patients with an alcohol consumption of more than 3 units/day did not have significantly different mortality than patients drinking occasionally,
HR $0.81(95 \%$ CI $(0.28$ to 2.35$) \mathrm{p}=0.70)$ and HR 1.51 (95\% CI (0.43 to 5.30$) \mathrm{p}=0.52$ ), respectively.

An analysis equal to the primary analysis, except that the reference group is set to ' $2-3$ units per day' (table 3 ), shows that this category has a significantly lower risk compared to 'One or less than 1 unit/day' (already seen in table 2), as well as a significantly lower risk than 'More than 3 units per day'.

Subgroup analyses showed no evidence of differential effects of alcohol intake between sex $(p=0.92)$, the presence or absence of comorbidity $(\mathrm{p}=0.76)$ or whether the patient lived alone or with the primary caregiver $(\mathrm{p}=0.78)$ (table 4). Omitting the subcategory 'Only at parties' from the analyses resulted in similar results as the primary analyses (table 4). Omitting the events in the first follow-up year from the analyses also gave results similar to the primary analyses (table 4 ).

The applicability of the Cox model was confirmed by a graphical check of the proportional hazard assumption in a Kaplan-Meier plot, as shown in figure 1 in the online supplementary appendix.

\section{DISCUSSION}

In this post hoc analysis, we found that in patients recently diagnosed with $\mathrm{AD}$, those who had a moderate alcohol intake (2-3 units/day) had a significantly lower risk of death compared with those who only had alcohol occasionally ( 1 or $<1$ unit/day), and with those who had high alcohol intake (more than 3 units/day). Abstinence or high alcohol intake did not significantly raise mortality compared with those drinking only occasionally.

Alcohol consumption was equal in the two RCT allocation groups; hence, alcohol consumption did not explain the mortality difference between the intervention and control groups.

To the best of our knowledge, we are the first to show an association between alcohol and mortality in patients with a diagnosis of AD. Previous studies have either focused on alcohol consumption and the risk of developing dementia, or on alcohol consumption and mortality in healthy subjects. ${ }^{17} 18$

Data in this study are disease specific for AD. This gives us important knowledge on how a very common lifestyle factor such as alcohol affects the lives of people diagnosed with $\mathrm{AD}$ specifically.

The association between alcohol and mortality in healthy subjects has been described as a U-shaped or J-shaped curve in earlier studies. ${ }^{17}{ }^{19}$ In this study in patients with dementia, we found that in abstinent patients and in patients with high alcohol intake there was no significant increase or decrease in mortality. An association that is suggestive of a protective effect between alcohol and mortality was seen only in the group of patients who had 2-3 units/day.

There are several possible explanations for the protective association between moderate alcohol intake and mortality observed in our study: (1) small amounts of 
Table 1 Baseline characteristics of patients with mild AD who participated in DAISY

\begin{tabular}{|c|c|c|c|c|c|}
\hline & $\begin{array}{l}\text { No alcohol } \\
n=25\end{array}$ & $\begin{array}{l}\leq 1 \text { Unit/day } \\
n=227\end{array}$ & $\begin{array}{l}2-3 \text { Units/day } \\
n=57\end{array}$ & $\begin{array}{l}\text { More than } 3 \text { units/day } \\
n=12\end{array}$ & $\begin{array}{l}\text { Test-p } \\
\text { value }\end{array}$ \\
\hline \multicolumn{6}{|l|}{ Sex } \\
\hline Male & $7(28)$ & $94(41.4)$ & $37(64.9)$ & 7 (58.3) & 0.0026 \\
\hline Female & $18(72)$ & $133(58.6)$ & $20(35.1)$ & $5(41.7)$ & \\
\hline Age & $76.4(73.5-78.5)$ & 77.4 (71.7-81.6) & 77.3 (71.9-80.03) & 75.3 (71.2-78.8) & 0.64 \\
\hline \multicolumn{6}{|l|}{ Awareness of disease } \\
\hline Full awareness & $7(28)$ & 72 (31.7) & $15(26.8)$ & $2(16.7)$ & 0.62 \\
\hline Shallow awareness & $14(56)$ & 130 (57.3) & 35 (62.5) & 10 (83.3) & \\
\hline No awareness & $4(16)$ & $25(11)$ & $6(10.7)$ & $0(0.0)$ & \\
\hline \multicolumn{6}{|l|}{ Smoking } \\
\hline Never & $11(44)$ & $62(27.3)$ & $15(26.3)$ & $1(8.3)$ & 0.26 \\
\hline Ex-smoker & $8(32)$ & 99 (43.6) & 30 (52.6) & 7 (58.3) & \\
\hline Smoker & $6(24)$ & $66(29.1)$ & $12(21.1)$ & $4(33.3)$ & \\
\hline MMSE & $24(22-26)$ & $24(22-26)$ & $24(22-26)$ & 23.5 (21.5-26.0) & 0.84 \\
\hline QoL-AD & $33(30-36)$ & $33(30-38)$ & 35 (29-39) & $34.5(27.5-40.5)$ & 0.70 \\
\hline ADSC-ADL & $65(55-70)$ & $65(55-72)$ & 61 (53-69) & 64.5 (53.0-69.5) & 0.47 \\
\hline \multicolumn{6}{|l|}{$\mathrm{CCl}$} \\
\hline 0 & $16(64)$ & $99(43.6)$ & $18(31.6)$ & $4(33.3)$ & 0.052 \\
\hline 1 & $7(28)$ & $90(39.7)$ & $26(45.6)$ & $8(66.7)$ & \\
\hline$>1$ & $2(8)$ & $38(16.7)$ & $13(22.8)$ & $0(0.0)$ & \\
\hline \multicolumn{6}{|l|}{ Household status } \\
\hline Living alone & $10(40)$ & $76(33.5)$ & $9(15.8)$ & $3(25)$ & 0.044 \\
\hline Living with others & $15(60)$ & $151(66.5)$ & 48 (84.2) & $9(75)$ & \\
\hline \multicolumn{6}{|l|}{ RCT allocation group } \\
\hline Control & $11(44)$ & $114(50.2)$ & $32(56.1)$ & $8(66.7)$ & 0.51 \\
\hline Intervention & $14(56)$ & $113(49.8)$ & $25(43.9)$ & $4(33.3)$ & \\
\hline \multicolumn{6}{|l|}{ Education } \\
\hline None & $7(28)$ & 97 (42.7) & $9(15.8)$ & $2(16.7)$ & \\
\hline Vocational/short & 8 (32) & 67 (29.5) & $9(15.8)$ & $2(16.7)$ & \\
\hline Medium/long & $10(40)$ & $63(27.8)$ & $39(68.4)$ & $8(66.7)$ & \\
\hline
\end{tabular}

Values are numbers (percentages) or median (IQR).

$\mathrm{p}$ Values where calculated with the Kruskal-Wallis test or the Pearson $\chi^{2}$ test.

ADSC-ADL, Alzheimer's disease Cooperative Study activities of daily living scale; CCl, Charlson Comorbidity Index; DAISY, The Danish Alzheimer Intervention Study; MMSE, Mini-Mental State Examination; QoL-AD, quality of life scale for Alzheimer's disease (proxy rated); RCT, randomised controlled trial.

alcohol may have a health preserving effect per se as indicated in studies in healthy subjects; (2) patients with moderate alcohol intake may have a richer social environment, which has been shown to improve the QoL and perhaps mortality; (3) the seemingly protective association may be caused by bias due to the fact that some patients with low intake may be in the terminal phase of their life.

In earlier studies, there are a variety of proposed explanations for the associations between moderate alcohol consumption and mortality in healthy subjects. Among these, evidence is perhaps strongest for

Table 2 Effect of alcohol consumption on mortality in patients with mild Alzheimer's disease (AD) with $\leq 1$ unit/day at baseline

\begin{tabular}{|c|c|c|c|}
\hline Alcohol consumption, units/day & $\begin{array}{l}\text { Number of deaths } \\
\text { (percentage of total number of } \\
\text { patients in the group) }\end{array}$ & $\begin{array}{l}\text { HR unadjusted } \\
(95 \% \mathrm{Cl})\end{array}$ & $\begin{array}{l}\text { HR adjusted* } \\
(95 \% \mathrm{Cl})\end{array}$ \\
\hline $\begin{array}{l}\text { No alcohol } \\
n=25\end{array}$ & $4(16)$ & $0.83(0.30$ to 2.30$)$ & 0.81 (0.28 to 2.35$)$ \\
\hline $\begin{array}{l}\leq 1 \\
n=227\end{array}$ & $42(18.5)$ & 1.0 (baseline) & 1.0 (baseline) \\
\hline $\begin{array}{l}2-3 \\
n=57\end{array}$ & $4(7.0)$ & $0.35(0.12$ to 0.98$)$ & $0.23(0.08$ to 0.68$)$ \\
\hline $\begin{array}{l}\text { More than } 3 \\
n=12\end{array}$ & $3(25.0)$ & $1.42(0.44$ to 4.57$)$ & $1.51(0.43$ to 5.30$)$ \\
\hline
\end{tabular}


Table 3 Effect of alcohol consumption on mortality in patients with mild Alzheimer's disease (AD) with 2-3 units/day at baseline

\begin{tabular}{|c|c|c|c|}
\hline Alcohol consumption, units/day & $\begin{array}{l}\text { Number of deaths } \\
\text { (percentage of total number of } \\
\text { patients in the group) }\end{array}$ & $\begin{array}{l}\text { HR unadjusted } \\
(95 \% \mathrm{Cl})\end{array}$ & $\begin{array}{l}\text { HR adjusted* } \\
(95 \% \mathrm{Cl})\end{array}$ \\
\hline $\begin{array}{l}\text { No alcohol } \\
n=25\end{array}$ & $4(16)$ & $2.35(0.59-9.40)$ & $3.50(0.84-14.62)$ \\
\hline $\begin{array}{l}\leq 1 \\
n=227\end{array}$ & $42(18.5)$ & $2.84(1.02-7.93)$ & $4.30(1.47-12.57)$ \\
\hline $\begin{array}{l}2-3 \\
n=57\end{array}$ & $4(7.0)$ & 1.0 (baseline) & 1.0 (baseline) \\
\hline $\begin{array}{l}\text { More than } 3 \\
n=12\end{array}$ & $3(25.0)$ & $4.02(0.90-17.98)$ & $6.50(1.38-30.68)$ \\
\hline
\end{tabular}

moderate alcohol consumption lowering mortality indirectly by decreasing the risks of cardiovascular disease. ${ }^{2}$ However, other plausible explanations such as modification of inflammation by lowering of interleukin 6 levels, ${ }^{20}$ or increase in insulin sensitivity, ${ }^{21}$ have been suggested.

Results concerning the effect of alcohol consumption on the development of dementia are diverging, but most studies have demonstrated a reduction in dementia and risk of $\mathrm{AD}$ with moderate alcohol intake compared to abstinence. ${ }^{422} 23$

It has been suggested that there are significant differences in subjects' abilities to metabolise alcohol, genetic differences being a determining factor for alcohol having either a protective or a harmful effect on cognitive abilities in later life. ${ }^{24}$ It should be considered whether this factor could be part of an explanation for the impact of alcohol on mortality in patients with $\mathrm{AD}$.

Regarding the importance of alcohol type when considering the risk of developing dementia, earlier studies have not been in agreement. Some have argued that only consumption of wine was associated with a lower risk of dementia, ${ }^{25}{ }^{26}$ while other studies observed no difference between types of alcohol. ${ }^{27} 28$

When using '2-3 units per day' as the reference group, we found that those with moderate alcohol consumption had a significantly lower risk of mortality than subjects with high alcohol intake. This points in the direction that increased alcohol intake is only protective until a certain consumption level.

Several limitations of our study must be acknowledged. The results of this study are based on post hoc analysis, that is, the investigation of alcohol intake on mortality was not described in the original DAISY protocol. ${ }^{7}$ However, the present research question was formulated (in an unpublished protocol) before the analyses were performed, and was not part of a portfolio of research questions. Hence, we believe that our results do not contain confirmation bias.

In the alcohol groups, other than our reference group ( 1 or $<1$ unit/day), the number of study participants was relatively low. The group 'More than 3 units per day' especially had few participants. This could be the reason for lack of power and therefore the lack of association between alcohol and mortality in the groups 'No alcohol' and 'More than 3 units per day'. However, the point estimates do not point towards an association.

In our questionnaires, we focused on the amount of alcohol consumed. We did not address other aspects of alcohol consumption that might be equally important. This includes considering if the group of abstainers contained both ex-drinkers and patients with lifelong

Table 4 Sensitivity and subgroup analyses

\begin{tabular}{|c|c|c|c|c|c|}
\hline & $\begin{array}{l}\text { No alcohol, } n=25 \\
\text { (HR 95\% Cl) }\end{array}$ & $\begin{array}{l}\leq 1 \text { unit/day, } \\
\mathrm{n}=227 \\
\text { (HR } 95 \% \mathrm{Cl} \text { ) }\end{array}$ & $\begin{array}{l}\text { 2-3 units/day, } \\
\text { n=57 } \\
\text { (HR 95\% Cl) }\end{array}$ & $\begin{array}{l}\text { More than } 3 \text { units/ } \\
\text { day, } n=12 \\
(\mathrm{HR} 95 \% \mathrm{Cl})\end{array}$ & $\begin{array}{l}\text { Test } \\
\left(p \text { value }{ }^{\star}\right)\end{array}$ \\
\hline Men & $0.46(0.06$ to 3.53$)$ & 1.00 (baseline) & $0.32(0.10$ to 0.96$)$ & $1.60(0.34$ to 7.50$)$ & 0.9170 \\
\hline Women & 1.07 (0.30 to 2.82$)$ & 1.00 (baseline) & 0.00 (no deaths) & $1.31(0.17$ to 10.44$)$ & \\
\hline No comorbidities & 1.26 (0.35 to 4.56$)$ & 1.00 (baseline) & 0.00 (no deaths) & $2.05(0.26$ to 16.46$)$ & 0.7595 \\
\hline Comorbidities & $0.36(0.05$ to 2.71$)$ & 1.00 (baseline) & $0.35(0.12$ to 1.06$)$ & $1.21(0.26$ to 5.70$)$ & \\
\hline Living alone & $0.44(0.055$ to 3.54$)$ & 1.00 (baseline) & $0.26(0.030$ to 2.21$)$ & $2.93(0.37$ to 22.94$)$ & 0.7765 \\
\hline Not living alone & $1.11(0.32$ to 3.80$)$ & 1.00 (baseline) & $0.23(0.066$ to 0.76$)$ & $1.21(0.26$ to 5.56$)$ & \\
\hline Omitting 'only at parties' & 1.23 (0.42 to 3.62$)$ & 1.00 (baseline) & $0.36(0.12$ to 1.10$)$ & 1.07 (0.22 to 5.27$)$ & \\
\hline First year events removed & $0.46(0.13$ to 1.62$)$ & 1.00 (baseline) & $0.14(0.040$ to 0.51$)$ & 0.99 (0.24 to 4.06$)$ & \\
\hline
\end{tabular}


abstinence and taking into account patterns of alcohol consumption. Since units were not divided into types of alcohol, we are unable to detect whether a possible protective association between moderate alcohol consumption and mortality is limited to one type of alcohol, for example, wine.

In this study, the amount of alcohol consumed by the patient was not self-reported, but was assessed by the primary caregiver and therefore less likely to be marred with inaccuracy caused by dementia. However, we cannot rule out that some patients took more alcohol than was noticed by their primary caregivers, or that the primary caregiver was not entirely accurate about the amount of alcohol a patient consumed. Characteristics of the caregivers might differ considerably between dyads (patient and primary caregiver). Cognitive abilities and the caregivers' own alcohol consumption levels are some of the things that are likely to have influenced both the actual amount of alcohol consumed by the patient and the amount reported.

We assessed alcohol consumption only once at inclusion. It might have been more representative to obtain measurements of alcohol consumption multiple times throughout the participant's lives after the $\mathrm{AD}$ diagnosis, thus characterising long-term alcohol consumption patterns for our patients with $\mathrm{AD}$ and possibly avoiding measurement errors.

The participants in this study make up a relatively homogeneous group of participants. They have been selected for an intervention study and there might therefore be an over-representation of better functioning patients. We analysed for some effect modification in a series of secondary analyses. Different effects of alcohol intake are generally expected between sexes because the official health authorities' guidelines on drinking are different for men and women based on the assumption of biological differences in ethanol metabolism proposed in earlier studies. In our analyses, no differing effects were seen.

It is an obvious concern that perhaps participants consuming alcohol moderately are generally healthier and consequently less likely to die in the follow-up period than those who do not consume alcohol.

To rule this out, we adjusted for premorbid conditions in our analysis by including CCI as a possible confounder. To accommodate any doubt whether the statistical correction fully controlled for differences in group composition concerning comorbidity, we performed a subgroup analyses. This showed no evidence of differential effects.

To rule out that the accuracy of ethanol reporting was questionable if a primary caregiver did not live together with the patient, a sensitivity analysis was performed that excluded patients living alone. Again, no differing effects were seen.

It can be speculated whether patients who are weak and approaching their terminal phase of life will naturally reduce their alcohol intake- a form of reverse causality. In this case, the protective effect that was found would be artificially inflated. To remove the worst cases of reverse causality, the first year of follow-up was removed from the analysis in a sensitivity analysis. This analysis retains the conclusions from the original primary analysis with effects that point in the same direction. This strengthens our belief that moderate alcohol intake is indeed associated with decreased mortality and not an artefact of reverse causation.

There might be concerns about the pattern of intake (binge drinking vs regular intake) in the groups 'Only at parties' and 'One or less than 1 unit/day', which was compiled under one group. However, omitting the subcategory 'Only at parties' from the analyses resulted in similar results as the primary analyses.

Since extensive data were collected on each patient in the DAISY study, we were able to adjust for a wide range of potential confounders, which is essential when studying the effects of alcohol consumption. However, there are a number of possible confounders that we did not adjust for. These include social status, body mass index, medication use and genetic factors such as apolipoprotein E. ${ }^{29}$

Other studies on alcohol and mortality have shown different curves for men and women. The non-significant interaction term between sex and alcohol intake found in our analysis indicates that the association between alcohol intake and mortality is similar for the two sexes and it is not necessary to stratify for sex in these analysis.

Guidelines on AD management advise against excessive alcohol consumption as a secondary preventive measure, ${ }^{30}$ but we have not been able to identify any guidelines concerning light-to-moderate alcohol consumption. Worldwide, approximately 35 million people currently suffer from dementia and the number is likely to increase considerably over the years to come. ${ }^{31} 32$

The WHO data from 2003 show that approximately $94 \%$ of the Danish population had consumed alcohol in the past 12 months. ${ }^{33}$ This shows us that there is likely to be a considerable number of people with $\mathrm{AD}$ who drink alcohol regularly. In view of that, more reflection should be given as to how alcohol affects subjects with $\mathrm{AD}$.

The results of our study point towards a potential, positive association of moderate alcohol consumption on mortality in patients with AD. However, we cannot solely on the basis of this study neither encourage nor advise against moderate alcohol consumption in patients with $\mathrm{AD}$.

Further studies are needed on this area. Studies on the effect of alcohol on cognitive decline and disease progression in patients with mild $\mathrm{AD}$ would be especially interesting.

Contributors SB drafted the manuscript and is the guarantor. SB, JK, VDS, GW and FBW outlined the statistical analysis. VDS conducted the statistical analysis. FBW and GW designed and conducted the DAISY study. All the authors assisted in the editing of this manuscript. All the authors read and approved the final manuscript.

Funding The DAISY study was supported by the National Board of Social Services at the Danish Ministry of Social Affairs, the Danish Ministry of Health and the Danish Health Foundation. 
Competing interests None declared.

Patient consent Obtained.

Provenance and peer review Not commissioned; externally peer reviewed.

Data sharing statement The data set is available from the corresponding author at sineberntsen@hotmail.com.

Open Access This is an Open Access article distributed in accordance with the Creative Commons Attribution Non Commercial (CC BY-NC 4.0) license, which permits others to distribute, remix, adapt, build upon this work noncommercially, and license their derivative works on different terms, provided the original work is properly cited and the use is non-commercial. See: http:// creativecommons.org/licenses/by-nc/4.0/

\section{REFERENCES}

1. Gronbaek M, Deis A, Becker U, et al. Alcohol and mortality: is there a U-shaped relation in elderly people? Age Ageing 1998;27:739-44.

2. Rimm EB, Klatsky A, Grobbee D, et al. Review of moderate alcohol consumption and reduced risk of coronary heart disease: is the effect due to beer, wine, or spirits? BMJ 1996;312:731-6.

3. Panza F, Frisardi V, Seripa D, et al. Alcohol consumption in mild cognitive impairment and dementia: harmful or neuroprotective? Int $J$ Geriatr Psychiatry 2012;27:1218-38.

4. Neafsey EJ, Collins MA. Moderate alcohol consumption and cognitive risk. Neuropsychiatr Dis Treat 2011;7:465-84.

5. Wiscott R, Kopera-Frye K, Seifert L. Possible consequences of social drinking in the early stages of Alzheimer disease. Geriatr Nurs 2001;22:100-4; quiz 05.

6. Phung KT, Waldorff FB, Buss DV, et al. A three-year follow-up on the efficacy of psychosocial interventions for patients with mild dementia and their caregivers: the multicentre, rater-blinded, randomised Danish Alzheimer Intervention Study (DAISY). BMJ Open 2013;3:e003584.

7. Waldorff FB, Buss DV, Eckermann A, et al. Efficacy of psychosocial intervention in patients with mild Alzheimer's disease: the multicentre, rater blinded, randomised Danish Alzheimer Intervention Study (DAISY). BMJ 2012;345:e4693.

8. Waldemar G, Waldorff FB, Buss DV et al. The Danish Alzheimer intervention study: rationale, study design and baseline characteristics of the cohort. Neuroepidemiology 2011;36:52-61.

9. American Psychiatric Association. Diagnostic and statistical manual of mental disorders. 4th edn. American Psychiatric Association, 1994

10. McKhann G, Drachman D, Folstein M, et al. Clinical diagnosis of Alzheimer's disease: report of the NINCDS-ADRDA Work Group under the auspices of Department of Health and Human Services Task Force on Alzheimer's Disease. Neurology 1984;34:939-44.

11. Pedersen CB. The Danish Civil Registration System. Scand J Public Health 2011;39(7 Suppl):22-5.

12. Folstein MF, Folstein SE, McHugh PR. "Mini-mental state." A practical method for grading the cognitive state of patients for the clinician. J Psychiatr Res 1975;12:189-98.

13. Logsdon R, Gibbons L. Quality of life in Alzheimer's disease: patients' and caregiver's reports. J Ment Health Aging 1999;5:21-32.

14. Galasko D, Bennett D, Sano M, et al. An inventory to assess activities of daily living for clinical trials in Alzheimer's disease.
The Alzheimer's Disease Cooperative Study. Alzheimer Dis Assoc Disord 1997;11(Suppl 2):S33-9.

15. Reed BR, Jagust WJ, Coulter L. Anosognosia in Alzheimer's disease: relationships to depression, cognitive function, and cerebral perfusion. J Clin Exp Neuropsychol 1993;15:231-44.

16. Charlson ME, Pompei P, Ales KL, et al. A new method of classifying prognostic comorbidity in longitudinal studies: development and validation. J Chronic Dis 1987;40:373-83.

17. Gronbaek M. The positive and negative health effects of alcoholand the public health implications. J Intern Med 2009; 265:407-20

18. Anstey KJ, Mack HA, Cherbuin N. Alcohol consumption as a risk factor for dementia and cognitive decline: meta-analysis of prospective studies. Am J Geriatr Psychiatry 2009;17:542-55.

19. Sadakane A, Gotoh T, Ishikawa S, et al. Amount and frequency of alcohol consumption and all-cause mortality in a Japanese population: the JMS Cohort Study. J Epidemiol 2009;19:107-15.

20. Wright CB, Sacco RL, Rundek T, et al. Interleukin-6 is associated with cognitive function: the Northern Manhattan Study. J Stroke Cerebrovasc Dis 2006;15:34-8.

21. Carlsson S, Hammar N, Grill V, et al. Alcohol consumption and the incidence of type 2 diabetes: a 20 -year follow-up of the Finnish twin cohort study. Diabetes Care 2003;26:2785-90.

22. Solfrizzi V, D'Introno A, Colacicco AM, et al. Alcohol consumption, mild cognitive impairment, and progression to dementia. Neurology 2007;68:1790-9.

23. Weyerer S, Schaufele M, Wiese B, et al. Current alcohol consumption and its relationship to incident dementia: results from a 3-year follow-up study among primary care attenders aged 75 years and older. Age Ageing 2011;40:456-63.

24. Ritchie SJ, Bates TC, Corley J, et al. Alcohol consumption and lifetime change in cognitive ability: a gene $\mathrm{x}$ environment interaction study. Age 2014;36:9638.

25. Nooyens AC, Bueno-de-Mesquita HB, van Gelder BM, et al. Consumption of alcoholic beverages and cognitive decline at middle age: the Doetinchem Cohort Study. Br J Nutr 2014:111:715-23.

26. Mehlig K, Skoog I, Guo X, et al. Alcoholic beverages and incidence of dementia: 34-year follow-up of the prospective population study of women in Goteborg. Am J Epidemiol 2008;167:684-91.

27. Ruitenberg A, van Swieten JC, Witteman JC, et al. Alcohol consumption and risk of dementia: the Rotterdam Study. Lancet 2002;359:281-6.

28. Mukamal KJ, Kuller LH, Fitzpatrick AL, et al. Prospective study of alcohol consumption and risk of dementia in older adults. JAMA 2003;289:1405-13.

29. Carmelli D, Swan GE, Reed T, et al. The effect of apolipoprotein E epsilon4 in the relationships of smoking and drinking to cognitive function. Neuroepidemiology 1999;18:125-33.

30. Health NCCfM. Dementia A NICE-SCIE Guideline on supporting people with dementia and their carers in health and social care. The British Psychological Society and Gaskell, 2007

31. World Health Organization. Fact sheet N362. Secondary Fact sheet N362 2012. http://www.who.int/mediacentre/factsheets/fs362/en/

32. Ferri CP, Prince M, Brayne C, et al. Global prevalence of dementia: a Delphi consensus study. Lancet 2005;366:2112-17.

33. World Health Organization. Global Health Observatory Data Repository Secondary Global Health Observatory Data Repository. 2013. http://apps.who.int/gho/data/node.main.A1044?lang=en 